\title{
Multimedia Transmission in Multi Hop Wireless Sensor Network
}

\author{
Sakthidharan G R \\ Research Scholar- CSE, \\ Anna university of Technology, \\ Coimbatore, India
}

\author{
S.Chitra \\ Phd, Principal, \\ Er.Perumal Manimekalai college of Engineering \\ and Tech., \\ Hosur, India
}

\begin{abstract}
Quality of Service (QoS) is a requirement for proper functioning of traditional and modern networks. Wireless Sensor Networks (WSN) is adversely affected because of mobility, harsh communication medium, and environment behavior. Quality of Service is a crucial aspect in WSN design information sent by nodes and delivered according to QoS requirements to provide outside observers accurate vision of monitored phenomena. In WSNs for effective transmission of still images, audio, and video information enforces rigorous necessities on the energy consumption and throughput. In this paper, it is proposed to investigate the performance of routing traffic for multimedia traffic in WSN for multihop nodes from the sink.
\end{abstract}

Keywords-Wireless Sensor Network, packet scheduling, Dynamic Source Routing, Multimedia traffic, one hop nodes.

\section{INTRODUCTION}

Technological advances in microelectronic mechanical systems (MEMS) and wireless communication technologies ensured the development of tiny, low-cost, low-power, and multifunctional smart sensor nodes in WSN. Wireless Sensor Networks (WSNs) are considered to be an important technology for the twenty-first century [1]. These sensor nodes are deployed and networked through internet and wireless links, providing opportunities for various civilian and military applications, like environmental monitoring, battle field surveillance, and industry process control [2]. Development of WSNs was first encouraged by military applications like battlefield surveillance, but they are now used in civilian applications like environment and habitat monitoring, healthcare applications, home automation, and traffic control. Differentiated from traditional wireless communication networks like cellular systems and mobile ad hoc networks (MANET), WSN's unique characteristics like dense node deployment level, sensor nodes higher unreliability and energy, computation, and storage constraints [3] provide new challenges in WSN development and application. Lot of research explored and solved various design/application issues, and major advances were made in their development and deployment.

A WSN consists of a huge number ranging from tens to thousands of low-cost, low-power, and multifunctional sensor nodes deployed in a region of interest. Such sensor nodes are small in size and equipped with embedded microprocessors, radio receivers, and power components to enable sensing, computing, communication, and actuation, the components being integrated on a single/multiple boards, and packed in space of a few cubic inches. WSNs communicate over short distances through wireless channels to share information and cooperative processing to complete common tasks. They are deployed globally for environmental monitoring and habitat study, over battlefields for military surveillance and reconnaissance, for search and rescue in emergency situations, for condition based maintenance and process control in factories, for infrastructure health monitoring in buildings and in homes to realize smart homes.

In a typical scenario, users retrieve interesting information from a WSN through queries and results from base stations (or sink nodes), which interact between users and network. Hence, WSNs are considered a distributed database. Sensor nodes are deployed inside the sink or close to it with limited power, computational capacity and memory. Sensor nodes are failure prone and may have global identification (ID) due to a huge overhead. They are also deployed in huge numbers. A WSN's fundamental goal is the production of information from local data got by individual sensor nodes by prolonging a WSN's life. Sensor nodes resource constrained nature poses special challenges to WSN design of WSNs for their applications. The limited power of sensor nodes necessitates designing an energy-efficient communication protocol. Sensor network routing is challenging as several factors distinguish them from other communication and wireless ad-hoc networks [4]. They are highly constrained with respect to transmission power, on-board energy, processing capacity, and storage requiring proper and careful management of resources. Researchers devised protocols for communication, and security in wireless networks, ad-hoc networks, mobile networks, etc. Much research was undertaken recently to investigate different aspects including low power protocols, network establishments, routing protocol, coverage problems and secure wireless sensor network establishment. Many protocols were suggested to prolong WSN life and to route correct data to sinks [5], [6], [7], [8]. Each method proposed in literature has its own advantages and disadvantages. Issues in WSN include limited battery power and low computational speed. Also, research targeted at specific applications needs to be undertaken. Thus, there is a need to study alternate and/or new protocols that ensures better and efficient use of scarce resources for applications at individual sensor nodes. 
As memory, bandwidth and processors are limited; QoS in WSN is a challenge. QoS policies should be simple and effective. The QoS policy design for WSN hardens as the network runs a big, distributed application. Earlier, in traditional networks, QoS centered on application differentiation like delay-sensitive traffic over delayinsensitive traffic. In WSN, QoS mechanisms centre on priorization (establishing an order) among an application's given tasks. Hence, highly specific requirements necessitate QoS techniques permitting applications to dictate own QoS policies. At present, literature pays scant attention to WSN QoS issues.

Usually, QoS is handled as first in a class of transmissions/application over others. In Link layer, access medium scheduling and the order in which packets should be sent are changed to meet QoS requirements. These can be achieved by packet reordering, priority control and admission policies. The amount of control packets can be adjusted to increase QoS of a single data packet. Packet priority is assigned by flow in wired networks, using packet queuing policies like token bus and WFQ (Weighted Fair Queuing) [9]. In WSN, there is no notion of flow which precludes per flow priorization. Due to link quality variability both number of copies sent and number of distinct routes for a specific packet is adjusted based on priority, increasing chances of successful packet delivery [10].

\section{QoS techniques}

Packet reordering: This algorithm category employs outgoing packet reordering to adjust spent time by queuing packets according to priority. The IEEE 802.11e standard separates packet queue in priority classes, defined by higher layers [11]. RAP (RealTime Architecture Protocol) employs packet reordering in WSNs [12]. With sensor nodes having resource restrictions, packets are divided among a few classes.

Medium access priorization and allocation: This algorithm category uses priorization policies which increases the probability of nodes with outgoing high priority packets in which the medium is accessed. In contention based protocols (such as 802.11e in EDCF - Enhanced Distributed Coordination Function - mode [11]), differentiated backoff policies and wait times are used for priority $\mathrm{f}$ every packet. In contention-free protocols like IEEE 802.15.4 in superframe mode, slots are ready for high priority packets [13]. WSN uses both mechanisms to effect latency requirements.

Admission control: These algorithms control data amounts where a node might send at a specific time. 802.11e uses admission control where a base station determines the number of bytes a station can forward in each superframe [11]. In WSN, admission control is sometimes implemented as distributed rate-adaptive MAC protocols. This signals the application which increase/decreases forwarded packet frequency [14].

Control overhead: These algorithms adjust control packet amount used for each data packet selectively to guarantee higher delivery. This increases delivery rates at lower bandwidth and higher energy consumption. According to packet priority, acknowledgment frames and RTS/CTS dialogs can be switched on/off. Also, transmissions that demand no loss like reprogramming tasks use control packets on each packet [10].

In the last ten years, there is a fast development in the sensor networking technologies. To provide detailed information about the hot spots in a large scale network, there is a necessity of high quality multimedia streams. In the recent sensor network structure, it is tough to enhance the multimedia streams with the restricted abilities of sensor node and sensor network. This paper proposes to investigate node performance for multimedia traffic which is one hop and multihop from a sink. The paper is organized as follows; section II reviews some of the related work available in the literature. Section III gives details about the experimental setup and results. Section IV concludes the paper.

\section{LITERATURE REVIEW}

Changsu Suh et al., [15] proposed an advanced IEEE 802.15.4 called TEA-15.4 that is based on traffic information, which adaptively regulates the active period of transmission. This TEA-15.4 utilizes two mechanisms Arbitrary Traffic Signal (ATS) and Traffic Time-Out (TTO) to identify the data traffic in the network. Using these two mechanisms the TEA-15.4 encourages multimedia communications by enough data throughput and also offers less energy consumption to sense device in WSNs. The full-standard IEEE 802.15.4 is employed on TinyOS to estimate the performance of the proposed TEA-15.4. Hence, TEA-15.4 is proved to be the apt mechanism for Wireless Multimedia Sensor Networks (WMSNs) observed from the results revealed from the test bed experiments and the TOSSIM simulator.

Abdul Hamidet al., [16] proposed a new QoS-aware routing protocol for WSN. The proposed protocol enhances high data rate for wireless multimedia sensor networks. The routing decision is performed with regard to the alteration of the necessary bandwidth dynamically and path-length-based on proportional delay demarcation for the real-time data. The functioning of this protocol is in a distributed manner in order to guarantee bandwidth and throughout delay necessities for real-time data. Simultaneously, an adjustment is made at the service rate of real-time and non-real-time data to improve the throughput of non-real-time data. In terms of average lifetime, average delay and network throughput the proposed protocol outperforms well that is illustrated by estimating the simulation results.

Min Chen et al., [17] proposed a new multipath routing method termed directional geographical routing (DGR), to deal the problem of real-time video streaming on a bandwidth and energy constricted wireless sensor network (WSN) by combining forward error correction (FEC) coding, the minute number of dispersed video-sensor nodes (VNs) to a sink. In order to transmit parallel FECprotected H.26L real-time video streams on a bandwidthlimited, undependable networking environment, the DGR develops an application-unique number of multiple 
disjointed paths for a VN. The fast packet delivery, load balancing and aggregation are assisted by the multiple paths in DGR. The performance of DGR is experimented through simulation on randomly generated WSNs that reveal the benefits: 1) considerably long network lifetime, 2) enhanced received video quality and 3) lower delay. Specifically, DGR enhances up to $3 \mathrm{~dB}$ the average video peak signal-to-noise ratio (PSNR) when compared to the other existing geographic routing methods.

Eren Gürses et al., [18] described the significant requirement that is a survey of the challenges faced in research and the exact position of literature in multimedia communication in WSN. The study focused mainly on the multimedia WSN applications, issues persuading multimedia delivery over WSN, solutions proposed recently in application, transport, and network layers, are indicated with the open research problems and their shortcomings. The main aim of this proposal is to provide a solution to develop efficient communication protocols and algorithms to realize multimedia WSN applications.

Mario Gerla et al., [19] proposed deployment of a limited number of mobile "swarms" in order to advance the sensor network. In terms of both networking capabilities and hardware functionalities, the swarm nodes possess improved capabilities than the sensor nodes. To the hot spots of the sensor network, the mobile swarms are directed to present the complete information about the intended area. With only low-cost high quality of multimedia streams are supported by the mobile swarms. To support the wireless backbone network for connecting various swarms and the routing methods a combined model is also described and is validated through simulation experiments.

In wireless multimedia sensor networks (WMSNs), to improve the potential for event description, the video sensors are employed. A single path is not able to provide the necessities of video transmission as the sensor nodes possess only limited transmission capacity. A multipath transmission is required subsequently. As the long routing path with a long end to end transmission delay will not gratify the time restriction of the video, therefore every path created by multipath routing algorithms is not appropriate for transmitting video. There are two types of information involved in each video stream: audio streams and image. The audio streams and image play roles differ and the importance levels also differ in different applications. To ensure the usage of limited bandwidth and energy in WMSNs, more priority must be provided to the significant stream (either audio stream or image stream). Lin Zhang et al., [20] proposed a Multi priority Multipath Selection (MPMPS) method in the transport layer to select the highest number of paths from every node disjoint found in routing paths to increase the outcome of streaming data transmission. The maximum number of paths for video transmission is efficiently selected by the MPMPS is illustrated through its simulation results.

Chenyang Lu et al., [12] proposed RAP for large-scale sensor networks, which is novel real-time communication architecture for WSN. For distributed micro-sensing applications, RAP ensures convenience, query at highlevel and event services. A light-weight and scalable network stack supports the new location-addressed communication prototypes. The velocity monotonic scheduling is a new packet scheduling policy that is introduced and estimated essentially for both distance and time limitations. This policy is specifically appropriate for communication scheduling in a large number of wireless devices that are integrated flawlessly into a physical space to execute real-time monitoring and control. The observation from the detailed simulation of RAP in representative sensor network environments reveals the decrease in the sensor network's end-to-end deadline miss ratio.

\section{EXPERIMENTAL SETUP}

The investigation of routing traffic was simulated in Opnet. The simulation setup consists of 28 wireless sensor nodes and one sink which was spread over an area of 4 sq. $\mathrm{Km}$. The nodes are spread over an area of $4 \mathrm{sq}$. Km, with the farthest node being 5 hops from the sink. The maximum available bandwidth is $11 \mathrm{Mbps}$ and with transmission power of each node is $0.005 \mathrm{w}$. Figure 1 shows the simulation environment, Node0, Node9, Node12 and Node24 are multihop away from the sink. Node4, Node6, Node15 and Node19 are the nearest to the sink and data is transmitted in one hop.

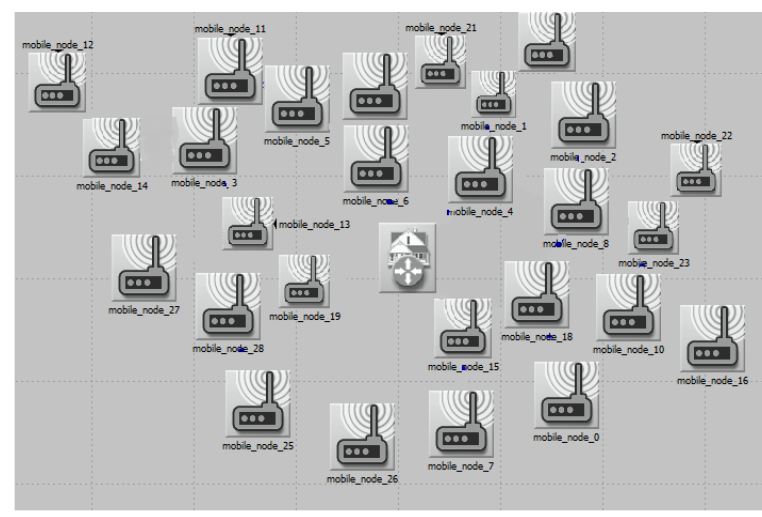

Figure 1: The experimental setup.

AODV routing protocol was used in this evaluation. The data transmitted from the nodes to the sink was high resolution video and random packet traffic. Simulations were carried out for 300 seconds. The network parameters of the one hop node and the $\mathrm{n}_{\max }$ hop node is studied. Figure 2 shows the routing traffic received by the one hop node and the $\mathrm{n}_{\max }$ hop node. From figure 2 it can be seen that nodes which are farthest away from the sink have lower control packet overheads resulting in lower utilization of power. The multihop nodes farthest away from the sink not only enjoy lower control packet overheads ( In this case about $20 \%$ of a single hop node) but also the overheads of routing are extremely low as they are not involved in the routing. 


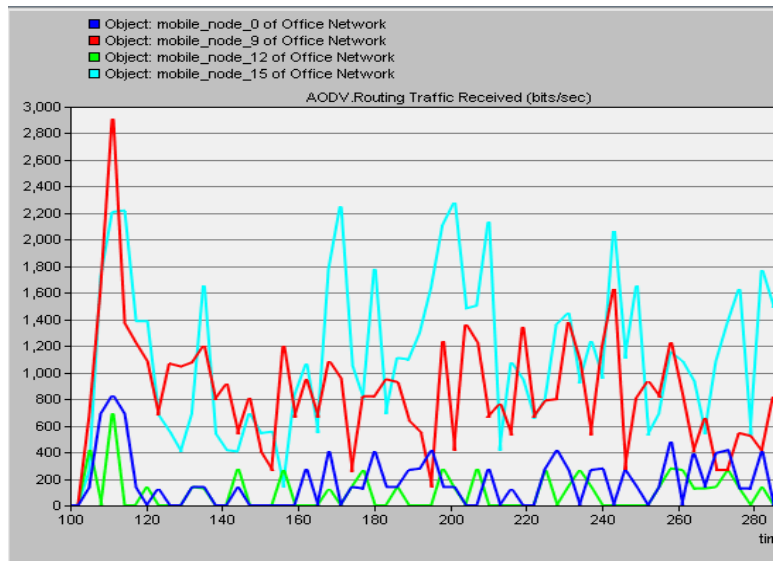

Fig 2: Routing traffic received by one hop and multihop node

Figure 3 shows the average routing traffic sent and received for the entire network.

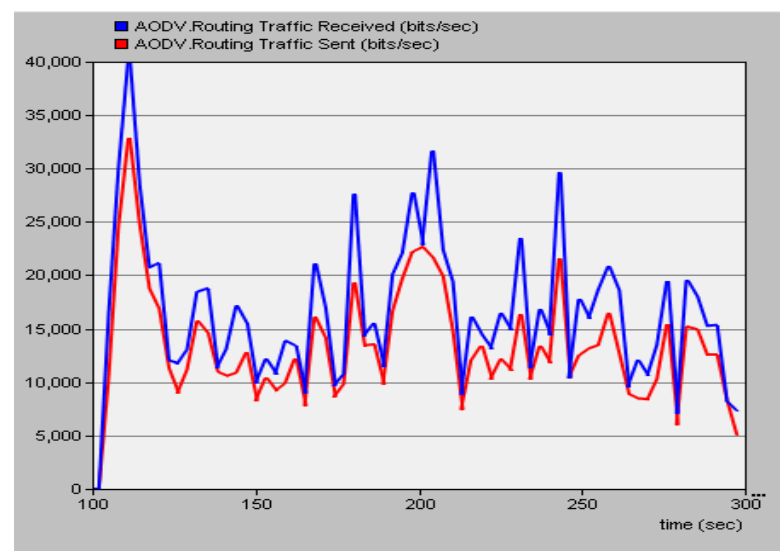

Figure 3: Routing traffic received and sent.

The path discovered from each WSN node to the sink is shown in Figure 4. From figure 4 it can be seen that most of the routes taken are the shortest path to the sink. The route discovery time is shown in Figure 5. The average time for route discovery with a maximum number of hops of 3 is around $3.5 \mathrm{~ms}$.

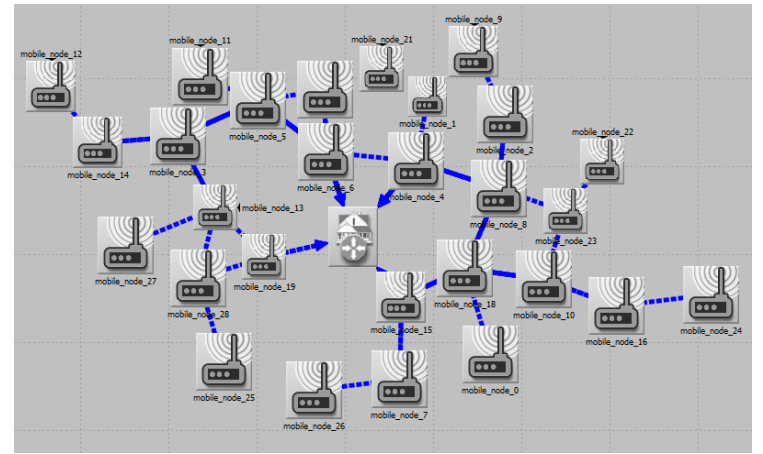

Figure 4: The routes discovered by AODV when all nodes are stationary.

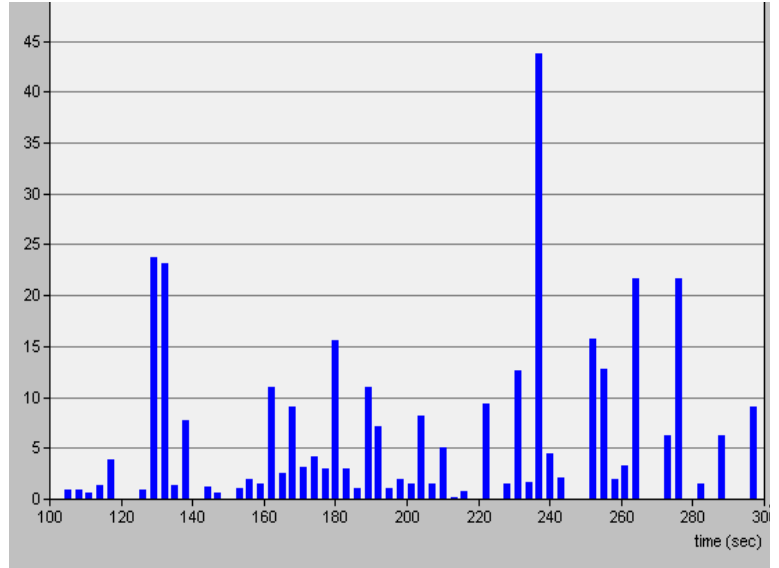

Figure 5: The route discovery time

The throughput of the $\mathrm{n}_{\max }$ single hop nodes are shown in figure 6 and figure 7 respectively.

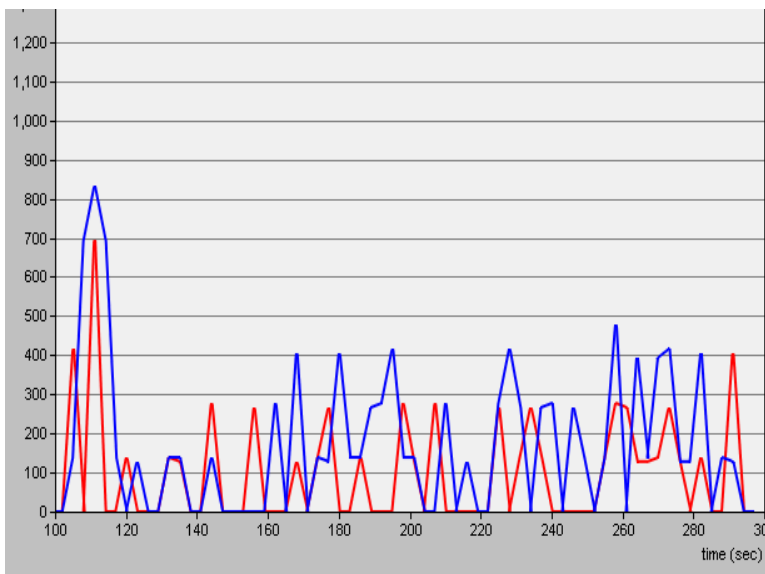

Figure 6: Throughput of two nodes farthest from the sink

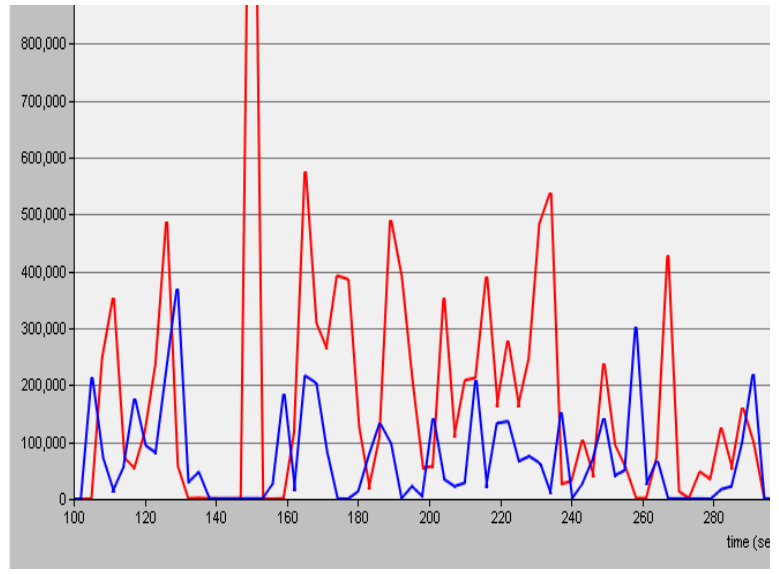

Figure 7: Throughput of two nodes closest to the sink 


\section{CONCLUSION}

In this paper it was proposed to investigate the effect of multimedia traffic in a multi hop wireless sensor network. The control packet overheads and the throughput were measured for the single hop nodes and multi hop nodes. It was observed that the control traffic overhead varies drastically with the increase in the control packet overheads by five fold in a single hop node. Further work needs to be proposed such to load balance the data flow so that the overall power dissipated from all the nodes are near uniform.

\section{REFERENCES}

[1] C.Y. Chong and S.P. Kumar, 2003, Sensor Networks: Evolution, opportunities, and Challenges, Proceedings of the IEEE, vol. 91, no. 8, pp. 12471256.

[2] Jun Zheng and Abbas Jamalipour, 2009, Wireless Sensor Networks: A Networking Perspective, a book published by A John \& Sons, Inc, and IEEE.

[3] I.F. Akyildiz, W. Su, Y. Sankarasubramaniam, and E. Cayirci, 2004 ,

A Survey on Sensor Network, IEEE Communication Magazine 40, 8 pp. 102-114.

[4] Kemal Akkaya and Mohamed Younis, A Survey on Routing Protocols for Wireless Sensor Networks.

[5] G. Martin, 2004, An Avaluation of Ad-hoc Routing Protocols for Wireless Sensor Networks, Master's thesis, School of Computing Science, Newcastle University upon Tyne, U.K.

[6] W.R. Heinzelman, A. Chandrakasan, and H. Balakrishnan, 2000 Energy-efficient Communication Protocol for Wireless Microsensor Networks, in IEEE Computer Society Proceedings of the Thirty Third Hawaii International Conference on System Sciences (HICSS '00), Washington, DC, USA,, vol. 8, pp. 8020 .

[7] W.R. Heinzelman, A. Chandrakasan, and $H$. Balakrishnan, 2002 An Application-Specific Protocol Architecture for Wireless Microsensor Networks in IEEE Tmnsactions on Wireless Communications, vol. 1(4), pp. 660-670.

[8] M.P. Singh, and M.M. Gore, A New Energy-efficient Clustering Protocol for Wireless Sensor Networks .

[9] Larry L. Peterson and Bruce S. Davie, 2003, Computer Networks: A Systems Approach, 3rd Edition. Morgan Kaufmann Publishers Inc.

[10] S. Bhatnagar, B. Deb, and B. Nath, 2001, Service differentiation in sensor networks. In Proceedings of the Fourth International Symposium on Wireless Personal Multimedia Communications.

[11] Yang Xiao. 2004, IEEE 802.11e: A QoS Provisioning at the MAC layer. IEEE Wireless Communications, 11(3):72-79.

[12] Chenyang Lu, Brian M. Blum, Tarek F. Abdelzaher, John A. Stankovic, and Tian He. RAP: A real-time communication architecture for large-scale wireless sensor networks. In Proceedings of the Eighth IEEE RealTime

[13] E. Callaway, P. Gorday, L. Hester, J.A. Gutierrez, M. Naeve, B. Heile, and V. Bahl, 2002, Home networking with IEEE 802.15.4: a developing standard for low-rate wireless personal area networks. IEEE Communications Magazine, 40(8):70-77.

[14] Alec Woo and David E. Culler. A transmission control scheme for media access in sensor networks. In Mobile Computing and Networking, pages 221235 ,

[15] Suh C, Mir Z, Ko Y 2008 Design and implementation of enhanced IEEE 802.15.4 for supporting multimedia service in wireless sensor networks. Comput Networks 52(13):2568- 2581.

[16] M. A. Hamid, M. M. Alam, and C. S. Hong , 2008, Design of a QoS-aware Routing Mechanism for Wireless Multimedia Sensor Networks, IEEE GLOBECOM 2008, New Orleans, LO, pp. 1-6.

[17] M. Chen, V. Leung, S. Mao, and Y. Yuan, 2007, Directional geographical routing for real-time video communications in wireless sensor networks, Comput. Commun., vol. 30, no. 17, pp. 3368-3383.

[18] E. Gurses, O.B. Akan, Multimedia communication in wireless sensor networks, Ann. Telecommun. 60 (78) (2005) 799-827.

[19] M. Gerla and K. Xu, 2003 Multimedia streaming in large-scale sensor networks with mobile swarms. SIGMOD Rec., 32(4):72-76.

[20] Zhang, L., Hauswirth, M., Shu, L., Zhou, Z., Reynolds, V., \& Han, G. 2008 . Multi-priority multipath selection for video streaming in wireless multimedia sensor networks. In LNCS: Vol. 5061. UIC'08:Proceedings of the 5th international conference on ubiquitous intelligence and computing (pp. 439-452). Berlin: Springer.

[21] Sakthidharan G.R., Chitra.S., 2012, A survey on wireless sensor network: An application perspective, IEEEE 